\title{
Optimum Plan for Step-down-stress Accelerated Life Testing with Censoring I and Numerical Simulation
}

\author{
Yu Wang ${ }^{1}$, Xiaoqin Zhang ${ }^{2}$ and Dianjun $\mathrm{Lu}^{1}$ \\ ${ }^{1}$ Department of Mathematics, Qinghai Normal University,Xining, 810008, China \\ ${ }^{2}$ Financial and Economic College, Qinghai University, Xining, 810016, China
}

\begin{abstract}
In this paper, we obtain the optimum plan by discussing a step-down-stress accelerated life testing (SDS-ALT) satisfying some specific condition at $k$ stresses under an exponential distribution.
\end{abstract}

Keywords-exponential distribution; step-down-stress accelerated life testing; accelerating life equation; numerical simulation

\section{INTRODUCTION}

Reliability evaluation for products of high-reliability and long-lifetime is one of the challenges in reliability engineering[1]. ZHANG Chun-hua[2-4] proposed a method of step-down-stress accelerated life testing (SDS-ALT). CHEN J and WANG D Y[5-6] did an experimental study on Double-crossed step-stress accelerated life testing. XU Xiao-ling, WANG Rong-hua and YU Song [7] studied step-down test based on lognormal distribution, and the finding of their study is that the efficiencies of step-down test is better than step-up test .WANG Yu-ming and SUN Yu-dong[8-9] studied double-crossed step-down-stress accelerated life test under different life distribution. LÜ Meng[10] gave an optimal design of double-crossed step-down-stress accelerated life test. Chernoff[11] initially discussed the optimum plans for simple constant-stress accelerated life tests under an exponential distribution, Mao shi song[12-13] studied constant-stress ALTs. J.F. Lawless and R. Liu [14-15] studied stress accelerated life testing . MEEKER W Q and AN Zong-wen [16-17] studied accelerated life problems based on certain distribution.

KOU Hai-xia and An Zong-wen[18] studied double synchronous-step-down-stress accelerated life testing .This paper gives the optimum plan for a step-down-stress ALT which satisfies the condition $\left\{\begin{array}{c}A_{1} \geq A_{2} \geq \cdots \geq A_{K} \\ \sum_{i=1}^{k} A_{i}=P, 0<P<1\end{array}\right.$ under an exponential distribution at $\mathrm{k} \geq 3$ constant stresses by minimizing the asymptotic variance of MLE in line with a linear accelerating equation .

\section{BASIC ASSUMPTION AND LEMMAS}

A step-down-stress ALT with censoring I can be designed as following:

We sample $n(n \geq r)$ products at random, supposing the unit number at stress level $S_{\mathrm{i}}$ to be $\tau_{1}$, divide them into $S_{2}$ groups and test the life times of the products at stress level $S_{1}$ respectively till $\tau_{2}$ products fail ,where $k-1$ is a usual stress level ,the failure data at stress level $S_{k}$ are denoted by $t_{i_{1}}, \ldots, t_{i_{r_{i}}}$ respectively $(\mathrm{i}=1, \ldots \mathrm{k})$. Let the life times of the products satisfy the following assumptions:

A1: The life times of the products at stress $S_{i}$ follow an exponential distribution with $F_{i}(t)=1-e^{-\lambda_{i} t}$, $T \geq 0, i=1, \ldots, k ; \theta_{i}=1 / \lambda_{i}$ is a mean life at stress $S_{i}$.

A2: The accelerating equation between mean life $\theta$ and stress $S$ is: $\ln \theta=a+b \phi(s)$, where $\phi(s)$ is a decreasing function of $S$.

According to the test data and the basic assumptions, the likelihood function is

$$
\delta_{i}=\theta_{i+1} \sum_{j=1}^{k} \frac{\tau_{j}-\tau_{j-1}}{\theta_{j}}, i=1,2 \ldots, k-1
$$

Where $\tau_{0}=0$ is the total time of the test at stress $S_{i}(\mathrm{i}=1, \ldots, \mathrm{k})$.

By (1.1) we have:

$$
L\left(\theta_{1}, \ldots \theta_{k} ; t_{11} \ldots, t_{1_{r 1}}\right)
$$

From A2, we have : $\theta_{i}=e^{a+b \Phi_{i}}$ and put it into (1.2), then

$$
\operatorname{LnL}(a, b)=-\sum_{i=1}^{k} r_{i}\left(a+b \Phi_{i}\right)-\sum_{i=1}^{k} T_{i} e^{-\left(a+b \Phi_{i}\right)}
$$

Lemma1.1: When testing with censoring I, we have $E\left(U_{i}\right)=n \theta_{i} p_{i} \prod_{j=0}^{i-1}\left(1-p_{j}\right), i=1,2, \cdots, k$

Among them 
$\tau_{0}=0, p_{0}=0, p_{i}=1-\exp \left(-\frac{\tau_{i}-\tau_{i-1}}{\theta_{i}}\right) i=1, \cdots, k \cdots$

Convenient for writing, make

$$
A_{i}=p_{i} \prod_{j=0}^{i-1}\left(1-p_{i}\right)
$$

\section{AsYMPTOTIC VARIANCE OF THE LOG MEAN}

To compute the Fisher information matrix of the log likelihood function (1.3)

$$
\begin{aligned}
& E\left[\frac{-\partial^{2} L n L(a, b)}{\partial a^{2}}\right]=\sum_{i=1}^{k} E\left(U_{i}\right) \cdot \exp \left[-\left(a+b \Phi_{i}\right)\right] \\
= & \sum_{i=1}^{k} n \theta_{i} p_{i} \prod_{j=0}^{i-1}\left(1-p_{i}\right) \\
& \exp \left[-\left(a+b \Phi_{i}\right)\right]=\sum_{i=1}^{k} n \theta_{i} p_{i} \prod_{j=0}^{i-1}\left(1-p_{i}\right) \theta_{i}^{-1} \\
= & \sum_{i=1}^{k} n p_{i} \prod_{j=0}^{i-1}\left(1-p_{i}\right)=\sum_{i=1}^{k} n A_{i}=A_{11} \\
E & {\left[\frac{-\partial^{2} L n L(a, b)}{\partial b^{2}}\right]=\sum_{i=1}^{k} \Phi_{i}^{2} E\left(U_{i}\right) \cdot \exp \left[-\left(a+b \Phi_{i}\right)\right] } \\
= & \sum_{i=1}^{k} \Phi_{i}^{2} n \theta_{i} p_{i} \prod_{j=0}^{i-1}\left(1-p_{i}\right) \theta_{i}^{-1}=\sum_{i=1}^{k} \Phi_{i}^{2} n \theta_{i} p_{i} \prod_{j=0}^{i-1}\left(1-p_{i}\right) \\
= & \sum_{i=1}^{k} \Phi_{i}^{2} n A_{i}=A_{22} \\
& E\left[\frac{-\partial^{2} \boldsymbol{L} n L(a, b)}{\partial a \partial b}\right]=\sum_{i=1}^{k} \Phi_{i} E\left(U_{i}\right) \theta^{-1} \\
& =\sum_{i=1}^{k} \Phi_{i} n \theta_{i} p_{i} \prod_{j=0}^{i-1}\left(1-p_{i}\right) \theta^{-1} \\
= & \sum_{i=1}^{k} \Phi_{i} n p_{i} \prod_{j=0}^{i-1}\left(1-p_{i}\right)=\sum_{i=1}^{k} \Phi_{i} n A_{i}=A_{12}=A_{21}(7)
\end{aligned}
$$

The following instructionsThe meaning of $n A_{i}$ : set $X$ For product life,The distribution of (1.3) so

$$
p\left(\tau_{i-1} \leq x<\tau_{i} \mid x>\tau_{i-1}\right)=\frac{p\left(\tau_{i-1} \leq \tau_{i}\right)}{p\left(x>\tau_{i-1}\right)}
$$

$$
\begin{aligned}
& =\left\{\left[1-\exp \left(-\frac{\delta_{i-1}+\tau_{i}-\tau_{i-1}}{\theta_{i}}\right)\right]-\left[1-\exp \left(-\frac{\delta_{i-1}}{\theta_{i}}\right)\right] / \exp \left(-\frac{\delta_{i-1}}{\theta_{i}}\right)\right\} \\
& =1-\exp \left(-\frac{\tau_{i}-\tau_{i-1}}{\theta_{i}}\right)=p_{i}
\end{aligned}
$$

Then the Fisher information matrix of the likelihood function is: $F=\left(\begin{array}{ll}A_{11} & A_{12} \\ A_{21} & A_{22}\end{array}\right)$, the inverse matrix of $\mathrm{F}$ is:

$$
\begin{gathered}
F^{-1}=1 / \Delta\left(\begin{array}{cc}
A_{22} & -A_{21} \\
-A_{12} & A_{11}
\end{array}\right) \\
\Delta=A_{11} A_{22}-A_{12}^{2}=\left(\sum_{i=1}^{k} r_{i}\right) \\
\left(\sum_{i=1}^{k} r_{i} \Phi_{i}^{2}\right)-\left(\sum_{i=1}^{k} r_{i} \Phi_{i}\right)^{2}=\sum_{j=2}^{k} r_{1} r_{j}\left(\Phi_{1}-\Phi_{j}\right)^{2} \\
+\sum_{j=3}^{k} r_{2} r_{j}\left(\Phi_{2}-\Phi_{j}\right)^{2}+\cdots+r_{k-1} r_{k}\left(\Phi_{k-1}-\Phi_{k}\right)^{2}
\end{gathered}
$$

And so the asymptotic variance of the log mean life at usual stress is :

$$
\begin{gathered}
\operatorname{As} \operatorname{Var}\left(\operatorname{Ln} \hat{\theta}_{0}\right)=\left(1, \Phi_{0}\right) F^{-1}\left(1, \Phi_{0}\right)^{\prime}= \\
1 / \Delta\left(A_{22}-2 A_{12} \Phi_{0}+\Phi_{0}^{2} A_{11}\right)=1 / \Delta\left(\sum_{i=1}^{k} r_{i} \Phi_{i}^{2}-2 \Phi_{0} \sum_{i=1}^{k} r_{i} \Phi_{i}\right. \\
\left.+\Phi_{0}^{2} \sum_{i=1}^{k} r_{i}\right)=\sum_{i=1}^{k} r_{i}\left(\Phi_{i}-\Phi_{0}\right)^{2} / \\
{\left[\sum_{j=2}^{k} r_{1} r_{j}\left(\Phi_{1}-\Phi_{j}\right)^{2}+\sum_{j=3}^{k} r_{2} r_{j}\left(\Phi_{2}-\Phi_{j}\right)^{2}+\cdots+r_{k-1} r_{k}\left(\Phi_{k-1}-\Phi_{k}\right)^{2}\right]}
\end{gathered}
$$

IV. OPtIMAL Distribution OF THE FAILURE NUMBER IN A STEP-DOWN-STRESS ALT WITH CENSORING I

To make computing and application easy, we take $\mathrm{k}$ accelerating stress levels satisfying:

$$
\left\{\begin{array}{l}
\Phi_{0}-\Phi_{1}=\mathrm{d} \Delta \\
\Phi_{i-1}-\Phi_{i}=\Delta
\end{array}\right.
$$

where $\mathrm{d}$ is a integer, $\Delta$ is a constant, $\mathrm{i}=2, \ldots, \mathrm{k}$.

Put (3.1) into (2.5), then: 


$$
\operatorname{As} \operatorname{Var}\left(\operatorname{Ln} \hat{\theta}_{0}\right)=\frac{\sum_{i=1}^{k} r_{i}(d+i-1)^{2}}{\sum_{\substack{i=1, \ldots, k-1 \\ j=2, \ldots, k \\ r<j}} r_{i} r_{j}(j-i)^{2}}=\frac{R}{S}
$$

The optimum test plan in a step-down-stress ALT , on one hand, requires the minimum Asymptotic variance of estimator at normal stress so as to improve the preciseness of statistical analysis, on the other hand ,needs to satisfy that the failure numbers at higher stress levels are not smaller than that at the minimum stress level so as to get more failure data in a shorten time, moreover, the following conditions should also be satisfied:

$$
\left\{\begin{array}{c}
A_{1} \geq A_{2} \geq \cdots \geq A_{K} \\
\sum_{i=1}^{k} A_{i}=P, 0<P<1
\end{array}\right.
$$

In the rest of the paper, we assume that (4.3) holds and give the optimal distribution plan of $\mathrm{r}$ failure numbers at $\mathrm{k}$ accelerating stress-levels by regarding the minimum asymptotic variance of the log mean as principle.

Theorem For a step-down-stress test with censoring I at $\mathrm{k}$ stress levels, if (3.3) holds, the optimum failure numbers of transformation are $A_{1}$ diminishing.

Proof:

$$
\begin{aligned}
& \sum_{i=1}^{k} A_{i}=p, \sum_{i=1}^{k} n A_{i}=n p \quad, n A_{k}=n p-n A_{1}-\cdots-n A_{k-1}, \\
& \frac{\partial n A_{k}}{\partial n A_{1}}=-1, \frac{\partial R}{\partial n A_{1}}=\frac{\partial}{\partial n A_{1}}\left[\sum_{i=1}^{k}(h+i-1)^{2} n A_{i}\right] \\
& =\frac{\partial}{\partial n A_{1}}\left[h^{2} n A_{i}+(h+1)^{2} n A_{2} \cdots(h+k-1)^{2} n A_{k}\right] \\
& =h^{2}-(h-k-1)<0(k>1) \\
& \frac{\partial S}{\partial n A_{1}}=\frac{\partial}{\partial n A_{1}}\left[n A_{1} n A_{2}+2^{2} n A_{1} n A_{3}+\cdots+(k-1)^{2} n A_{1} n A_{k}+n A_{2} n A_{3}\right] \\
& \left.=1^{2} n A_{2}+2^{2} n A_{3}+\cdots+(k-2)^{2} n A_{2} n A_{k}+\cdots+n A_{k-1} n A_{k}\right] \\
& -\cdots(k-1)^{2} n A_{k}-(k-1)^{2} n A_{1} \\
& =-(k-1)^{2} n A_{1}-\left[(k-2)^{2}-1^{2}\right] n A_{2}-\cdots-\left[\left[1^{2}-(k-2)^{2}\right] n A_{k-1}+(k-1)^{2} n A_{k}\right]
\end{aligned}
$$

$$
\begin{aligned}
& \frac{\partial S}{\partial n A_{1}}=(k-1)^{2}\left(n A_{k}-n A_{1}\right)+\left[(k-2)^{2}-1^{2}\right]\left(n A_{k-1}-n A_{2}\right) \\
& +\cdots+\left[\left(\frac{k}{2}\right)^{2}-\left(\frac{k}{2}-1\right)^{2}\right]\left(n A_{\frac{k}{2}+1}-n A_{\frac{k}{2}}\right) \\
& =\sum_{i=1}^{k / 2}\left[(k-i)^{2}-(i-1)^{2}\right]\left(n A_{K-i+1}-n A_{i}\right) \quad \text { from } \quad(3.3) \\
& A_{1} \leq A_{2} \leq \cdots \leq A_{k} \\
& i=1,2, \cdots, k / 2,(k-i)^{2}-(i-1)^{2}>0 \\
& \frac{\partial S}{\partial n A_{1}}=(k-1)^{2}\left(n A_{k}-n A_{1}\right)+\left[(k-2)^{2}-1^{2}\right]\left(n A_{k-1}-n A_{2}\right) \\
& +\cdots+\left[\left(\frac{k+1}{2}\right)^{2}-\left(\frac{k-3}{2}\right)^{2}\right]\left(n A_{(k+3) / 2}-n A_{(k-1) / 2}\right) \\
& +\left[\left(\frac{k-1}{2}\right)^{2}-\left(\frac{k-1}{2}\right)^{2}\right] n A_{(k+1) / 2} \\
& \\
& +(k-1) / 2 \\
& =\sum_{i=1}^{2}\left[(k-i)^{2}-(i-1)^{2}\right]\left(n A_{k-i+1}-n A_{1}\right) .
\end{aligned}
$$

From (3.3)to $A_{1} \leq A_{2} \leq \cdots \leq A_{k}$. When $i \leq(k-1) / 2$,

$$
\begin{aligned}
& (k-i)^{2}-(i-1)^{2}>0 n A_{k-i+1}-n A_{i} \geq 0, \frac{\partial S}{\partial A_{1}} \geq 0, \frac{\partial S}{\partial A_{n}} \geq 0, \\
& R>0, S>0
\end{aligned}
$$$$
\frac{\partial \operatorname{Asvar}\left(\ln \hat{\theta}_{0}\right)}{\partial n A_{1}}=\left(\frac{\partial R}{\partial n A_{1}} S-\frac{\partial R}{\partial n A_{1}} R\right) / S^{2}<0
$$

By the type known $\partial A s \operatorname{var}\left(\ln \hat{\theta}_{0}\right)$ about $A_{1}$ Strictly decreasing and decreasing is independent of the value of $A_{k}$.

\section{Optimal Distribution of the SAMple Number IN A STEP-DOWN-STRESS ALT WITH CENSORING I}

One of the purposes to accelerate life tests is to shorten the testing time and cut down the testing cost ,therefore ,one can assume that the mean times of the step-down-stress SDS-ALT at time $\tau$ geometric sequence. $A_{1}=A_{2}=\cdots=A_{k}=\frac{P}{K}$. $A_{i}=A_{i-1}, i=2, \cdots k$ and $A_{i}=\left(1-p_{1}\right) \cdots\left(1-p_{i-1}\right) p_{i}$ 


$$
\begin{aligned}
& \left(1-p_{1}\right) \cdots\left(1-p_{i-2}\right)\left(1-p_{i-1}\right) p_{i}=\left(1-p_{1}\right) \cdots\left(1-p_{i-2}\right) p_{i-1} \text { so } \\
& p_{i}-\frac{p_{i-1}}{1-p_{i-1}}, \text { one ore } p_{i}=\exp \left(-\frac{\tau_{i}-\tau_{i-1}}{\theta_{i}}\right) \\
& 1-e-\frac{\tau_{i}-\tau_{i-1}}{\theta_{i}}=\left(1-e-\frac{\tau_{i-1}-\tau_{i-2}}{\theta_{i-1}}\right) / e-\frac{\tau_{i-1}-\tau_{i-2}}{\theta_{i-1}} \text { Taylor } \\
& =e-\frac{\tau_{i-1}-\tau_{i-2}}{\theta_{i-1}}-1
\end{aligned}
$$

show

$$
\begin{aligned}
& e^{x} \approx 1+x, 1-\left(1-\frac{\tau_{i}-\tau_{i-1}}{\theta_{i}}\right)=\left(1+\frac{\tau_{i-1}-\tau_{i-2}}{\theta_{i-1}}\right)-1 \\
& \text { So, } \frac{\tau_{i}-\tau_{i-1}}{e^{b \Phi_{i}}}=\frac{\tau_{i-1}-\tau_{i-2}}{e^{b\left(\Phi_{i}+d\right)}}, \frac{\tau_{i}-\tau_{i-1}}{\theta_{i}}=\frac{\tau_{i-1}-\tau_{i-2}}{\theta_{i-1}} \\
& \frac{\tau_{i-1}-\tau_{i-2}}{\tau_{i}-\tau_{i-1}}=e^{b d}, i-2, \cdots k
\end{aligned}
$$

\section{ACKNOWLEDGEMENT}

This research was financially supported by Science and Technology Innovation Ability Enhancement Planning Project of Qinghai Province (2015-ZJ-724) and by the Ministry of Education Chunhui Program JWSL[2014]1310.

\section{REFERENCES}

[1] TAN Wei, SHI Yi-min, SUN Yu-dong, Reliability simulation for step-down-stress accelerated life testing, Computer Simulation, 2011, 28(12): 80-83.

[2] ZHANG Chun-hua, CHEN Xun, WEN Xi-sen, Step- down-stress accelerated life test-methodology Acta Armamentarii, 2005, 26(5): 661-665.

[3] ZHANG Chun-hua, CHEN Xun, WNE Xi-sen, Step-down-stress accelerated life test-statistical analysis, Acta Armamentarii, 2005, 26(5): 666-669.

[4] WANG Ya-shun, ZHANG Chun-hua, CHEN Xun, Step- down-stress accelerated life test-optimal design, Acta Armamentarii, 2007, 28(6): 686-691.

[5] CHEN J, WANG D Y, FU Y L, et al. Double-crossed step-stress accelerated life testing for pneumatic cylinder, Applied Mechanics and Materials, 2012, 121-126: 1274-1278.

[6] CHEN J, LI J, WANG D Y, et al. Double crossed step-down-stress accelerated life testing for pneumatic cylinder based on cumulative damage model, Advanced Materials Research, 2014, 871: 56-63.

[7] XU Xiao-ling, WANG Rong-hua, YU Song, et al. Comparison analysis of efficiencies for lognormal distribution based on step acceleration test and step deceleration test, Journal of Mechanical Engineering, 2009, 45(4): 56-63.

[8] WANG Yu-ming, CAI Jin-yan. Numerical simulation of double-cross-step-down-stress accelerated life test, Computer Simulation, 2008, 25(12): 342-345.

[9] SUN Yu-dong, SHI Yi-min, Numerical simulation of double-crossed step-down-stress accelerated life test under lognormal distribution, Fire Control \& Command Control, 2012, 37(9): 23-26.
[10] LÜ Meng, CAI Jin-yan, PAN Gang, et al. Optimal design of double-crossed-step-down-stress accelerated life test based on Monte-Carlo simulation, Electronics Optics \& Control, 2013, 20(10): 96-101.

[11] Chernoff H., Optimal accelerated life designs for estimation, Tech nometrics ,vol. .4 , No.3 1962.

[12] S .Mao and Yo Cheng, Improvement of the data analysis in constant ,stress ALT ,J. Fujian normal university ,No2:23-28,1982.

[13] S .Mao ,Bayesian method of constant-stress ALT, J. Appl. Probability and statistics . No 3: 264-268, 1987.

[14] J. F. Lawless, Statistical models and methods in life data ,statistics press of China, 1998.

[15] R. Liu, Optimal plan for constant-stress ALT ,J .East China Normal University No. 4, 2000.

[16] MEEKER W. Q., ESCOBAR L. A., A review of recent research and current issues in accelerated testing, International Statistical Institute(ISI), 1993, 61(1): 147-168.

[17] AN Zong-wen, HUANG Hong-zhong, WANG Gui-bao, Accelerated life mode based on bivariate exponential conditional distribution, Journal of University of Electronic Science and Technology of China, 2009, 38(3): 455-458.

[18] KOU Hai-xia, An Zong-wen, Liu Bo, Double Synchronous-Step-Down-Stress Accelerated Life Testing Method, Journal of University of Electronic Science and Technology of China 2016(2): 316-320. 